\title{
Selective Retina Therapy
}

\author{
Boris Považay, Ralf Brinkmann, Markus Stoller, \\ and Ralf Kessler
}

\subsection{Retinal Therapy: A Short Historic Overview}

Retinal light based therapy has been discovered in the late 1940s by using sunlight [1] which was soon replaced by Xenon light flashes, and eventually by coherent monochromatic radiation, once powerful lasers became available. The latter first proved successful coagulation in clinical trials in the early 1970s [2].

Traditional laser photocoagulation (LPC) utilizes thermal interaction by which light energy absorbed by tissue pigments is converted to heat, thus causing photothermal denaturation whereas the temperature rise is dependent on laser power, wavelength and exposure time, in addition to the properties of the target tissue [3]. LPC techniques have been a mainstay for the treatment of several

B. Považay

HuCE optoLab, Bern University of Applied Sciences, Biel, Switzerland

\section{R. Brinkmann $(\bowtie)$}

Institute of Biomedical Optics, University of Lübeck, Lübeck, Germany

Medical Laser Center Lübeck, Lübeck, Germany

M. Stoller

Meridian AG, Thun, Switzerland

R. Kessler

Heidelberg Engineering GmbH, Heidelberg,

Germany retinal diseases. Multiple hypotheses regarding the biological effects of the different diseases are still in discussion, including reduction of oxygen demand by destroying photoreceptors, alterations of the local permeability and laser stimulation of RPE in edematous areals for proliferation and rejuvenation. Independent of the lack of a full understanding of the underlying mode of action, LPC was found beneficial to prevent retinal detachment after retinal hole formation [1], for the treatment of diabetic retinopathy (DR) [4], diabetic macular edema (DME) [5], neovascular age-related macular degeneration (AMD) [6], for central serous retinopathy (CSR) [7] and some other retinal pathologies.

LPC has been shown to be very successful in panretinal photocoagulation for DR hypothesizing that a reduction in oxygen demand by coagulating retinal tissue in the periphery preserves the central vision. Further, a macular grid treatment for DME was and still is very successful with respect to maintaining vision and reducing oedema in the central macula.

Laser therapy for AMD has an even more changeful history that was driven by the availability of laser technology and diagnostic tools. Starting in the 1970s, laser parameters used in early studies up to the late 1990s and even at the beginning of the new millennium were widely inconsistent. Reports on improvements as well as lack of correlation in respect to the treatment 
can be found. In most cases, researchers utilized readily available laser diodes or argon ion lasers with the ability to irradiate tissue in continuous or quasi continuous wave $(\mathrm{cw})$ irradiation modes. As most recently reviewed in [8] studies involved fellow-eye treatment of drusen sites and their short-term visibility as well as long-term changes. Indicating either slightly more positive or negative outcomes for AMD, the inconsistency in the results might be associated with different levels of choroidal neovascularization due to inflammatory response caused by necrosis of RPE and neighbouring tissue. LPC posed an important tool before the advent of a wide range of pharmaco-therapeutical agents that diminished the significance of laser-treatment to timecritical cases. Despite the drugs' success, several clinical trials indicate that therapeutic effects of intravitreal agents such as anti-VEGF injections and steroid implants are short-term compared to those of traditional laser photocoagulation therapy (LPC) and furthermore have the drawback that their application cannot be precisely controlled spatially [9]. To avoid regular arduous, cost-intensive and also risky injections required for chemical treatment of the chronic and recurrent disease, improvements in laser irradiation were pursued.

Due to the slow heating process and the strong heat dissipation into the surrounding tissue when using millisecond laser exposure, massive collateral damage of surrounding components like Bruch's membrane and choriocapillaris as well as the particularly healthy overlying photoreceptor (PR) cells is unavoidable $[10,11]$. These adverse effects can lead to scotoma, reduced night vision, and disruption of the retinal anatomy through scarring $[12,13]$.

In the last decade, microsecond pulsed lasers in the green spectral range have become readily available for clinical applications that try to induce a specific therapeutic effect, but avoid damage of the PR, neural retina and choroid by selective targeting of different fundus structures. The following sections describe the laser effects on the RPE layer, the idea of its selective treatment, the underlying physics as well as initial experiments and clinical study results.

\subsection{The Concept and State of the Art of Selective Retina Therapy}

The retinal pigment epithelium (RPE) is a monocellular layer located at the outer retina between the photoreceptors on its apical side and Bruch's membrane adjacent on its basal side (Fig. 11.1a). The RPE cells are connected with tight junctions and thus forming the blood-retina barrier to the choroid. The RPE has multiple tasks [14]; upon many others it controls the outer retinal metabolism and continuously pumps water diffusing from the vitreous body into the retina towards the choroid, in order to prevent retinal swelling. The cells apical microvilli encompass the rear parts of the rods and cones outer segments, which are frequently shed and phagocytosed within the RPE cells to regenerate 11-cis-retinaldehyde serving as the visual pigment crucial in the vision cycle.
Fig. 11.1 Transmission electron microscopies of a young (a) [18] and an (70 years) old human RPE cell (b) [16]. $z k$ nucleus, $p g$ pigment (melanosome), as photoreceptor outer segment, bm Bruch's membrane, $L$ lipofuscin (partly comprising pg at arrowheads)
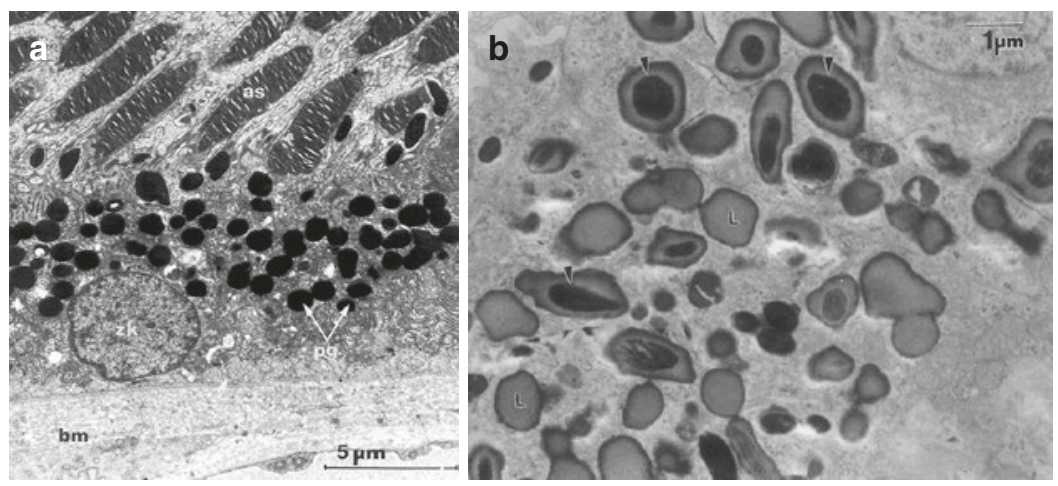
Metabolic, lipid rich end products emerging from this process are supplied toward the choroid. With respect to molecular transport of proteins, oxygen and water, the RPE and Bruch's membrane represent a diffusion barrier. With increasing age, RPE cells thicken and become heavily loaded with metabolic fatty end products like lipofuscin resulting from the lysosomal degradation pathways (Fig. 11.1b) [15, 16]. With the concomitant thickening of Bruch's membrane, eventually the water transport to and nutrition transport from the choroid is heavily compromised [17].

For a variety of retinal diseases, especially those which are thought to be associated with a degradation and reduced metabolism at the retina, it might be elegant to selectively eliminate specific regions of the monocellular RPE layer and thereby trigger its regeneration by wound healing. It is known that the RPE is able to rapidly close small wounds as found after mild photocoagulation. Such a cellular rejuvenation might lead to an improved metabolism, molecular transport and retinal functionality. However, the adjacent highly sensitive neural retina and choroid should not be affected by such a process.

The question arose if and how such a selective RPE treatment can be induced. In 1983 Anderson and Parrish published the idea of selective photothermolysis based on the concept of an absorption contrast with a stronger light absorption in the target area embedded in a less absorbing surroundings [19]. Selective treatment is postulated when using repetitive laser pulses with peak temperatures allowing sufficient denaturation rates just at the absorbing sites. This concept is perfectly suited for the RPE cells being heavily loaded with strongly light absorbing melanosomes (Fig. 11.1: 100-200 per cell, each about $1 \mu \mathrm{m}$ in size). About $20-80 \%$ of light in the green spectral region is absorbed by the RPE [15]. Melanosome distribution and absorption varies inter- and intraindividually. It typically increases towards the periphery and decreases with age [20].

In order to selectively heat and damage the RPE only, without affecting the surroundings, heating should take place far below the RPE's thermal confinement time, defined as decreasing of the maximum temperature rise to $36 \%(1 / \mathrm{e})$.
When assuming a $3 \mu \mathrm{m}$ uniformly absorbing sheet, neglecting the individual melanosomes, and a much larger heating spot diameter, then about $30 \mu$ s are estimated. Thus using heating times far below $30 \mu$ s should enable strong temperatures at the RPE with strong temperature gradients towards the much less absorbing environment [21]. Such a quick heating can be obtained with high power laser pulses, preferentially at wavelengths in the green spectral range due to high melanin absorbance. Unfortunately, $\mu$ s heating time requires very high irradiance and thereby laser power in order to obtain temperatures for a sufficient thermal damage rate. According to the Arrhenius theory describing thermal damage as a first order rate process [3], a $30 \mu$ s single pulse already requires temperatures exceeding $100{ }^{\circ} \mathrm{C}$, when taking the commonly used Arrhenius constants for retinal denaturation into account. However, as thermal damage is accumulative, applying multiple pulses with lower damage rates was assumed to be also sufficient, provided that an appropriate cooling time between the pulses is met in order not to lose selectivity owing to background heating by thermal diffusion.

\subsubsection{Experimental Results}

First promising results on such selective photocoagulation of the RPE by means of a high power chopped argon ion laser at $514 \mathrm{~nm}$ with a pulse duration of $5 \mu \mathrm{s}$ and a repetition rate of $500 \mathrm{~Hz}$ on rabbits utilizing a $110 \mu \mathrm{m}$ spot diameter have been published by Roider et al. [21]. In order to achieve the required irradiance an increase of the necessary power for larger spots and shorter pulses, a frequency-doubled and Q-modulated $\mathrm{Nd}$ :YLF laser with intracavity second harmonic generation to a wavelength of $527 \mathrm{~nm}$ was developed, which allowed to adjust the pulse duration between 250 ns and $3 \mu$ s with almost constant peak power and pulse energies around $1 \mathrm{~mJ}$ [22]. The laser was used to investigate the damage thresholds depending on the pulse energy and duration as well as the number of pulses on porcine RPE explants ex vivo by means of a vitality stain. Figure 11.2 shows that the effective dose 
for $50 \%$ probability $\left(\mathrm{ED}_{50}\right)$ thresholds of cell damage increases from $120 \mathrm{~mJ} / \mathrm{cm}^{2}$ at $250 \mathrm{~ns}$ almost linearly with pulse duration up to $220 \mathrm{~mJ} /$ $\mathrm{cm}^{2}$ for $3 \mu \mathrm{s}$ for single pulses. Increasing the number of pulses reduces the threshold radiant exposure per pulse by $50 \%$ (250 ns pulse) and $20 \%$ ( 1 and $3 \mu \mathrm{s})$ with a saturation observed at around 500 pulses, respectively [22], although the accumulated radiant exposure is increased within the exposure region.

As an additional concept, a continuous wave argon ion laser beam was used in a rapid scanning mode also providing $\mu$ s illumination times. Using an $19 \mu \mathrm{m}$ laser spot diameter at a scanning speed of $11.7 \mathrm{~m} / \mathrm{s}$ in order to achieve a $1.6 \mu \mathrm{s}$ irradiation time, a threshold power of $569 \mathrm{~mW}$ was found for ten repetitive scans, which corresponds to a threshold radiant exposure of $297 \mathrm{~mJ} /$ $\mathrm{cm}^{2}$ [23]. Thresholds found here were slightly higher than with the pulsed application, but also showed saturation for multiple hundred scans
(Fig. 11.2) [23]. The technique was approved on rabbits showing the desired RPE effects [24]. Interestingly, the retinal spot scanning speed of Heidelberg Engineering's confocal scanning laser ophthalmoscopes (cSLO) is around $70 \mathrm{~m} / \mathrm{s}$ which corresponds to a single spot irradiation time of about $270 \mathrm{~ns}$. This irradiation time had already been proved with a pulsed laser in clinical trials [25] and thus a cSLO represents another promising irradiation modality for SRT.

However, the threshold dependence on the pulse duration and the saturation with higher number of pulses found were in contradiction to the thermal damage theory of Arrhenius. Thus other mechanisms of cell damage had been taken into account. Lin et al. showed selective damage of artificially pigmented cells by microbubble formation (MBF) around phagocytosed absorbers induced by Q-switched laser pulses [26]. Microbubble occurrence always coincided with immediate cell death observed with a vitality stain. In order to

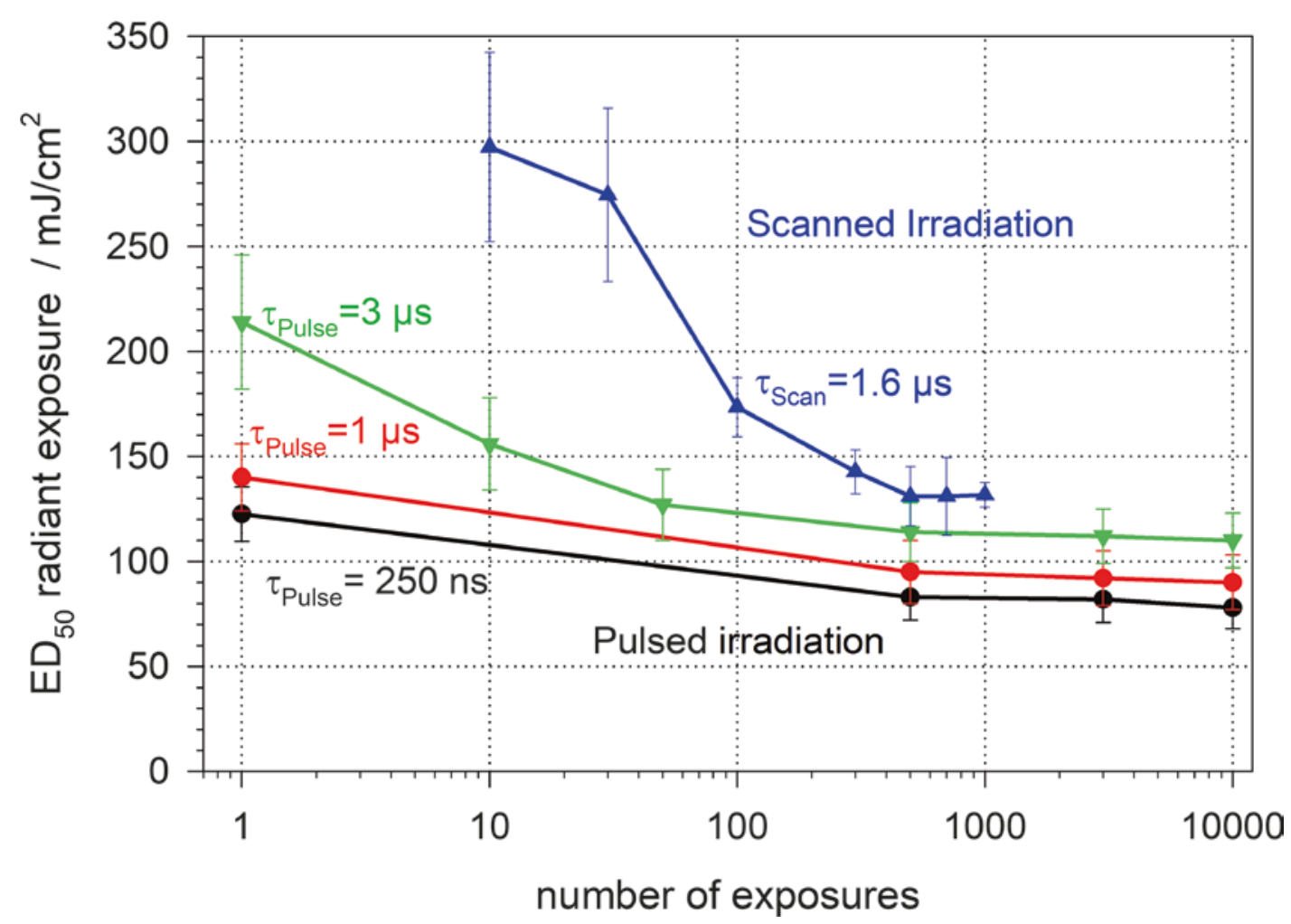

Fig. 11.2 Threshold radiant exposure for RPE cell damage on the number of pulses applied for different irradiation modalities and parameters $[22,23]$ 
understand MBF closer, the melanosomes and the granular structure of the absorbers needed to be taken into account. The thermal confinement time for a $1 \mu \mathrm{m}$ diameter melanosome can be estimated to about $450 \mathrm{~ns}$ [27]. Microbubbles nucleate heterogeneously at the surface of the melanosomes and then grow around the melanosomes [27]. Investigations on the bubble dynamics revealed a growth of the bubbles proportional to the radiant exposure for ns pulse duration, but an oscillation with limited maximal size of about $4 \mu \mathrm{m}$ in a selflimiting process when using $\mu$ s pulse durations [28]. Investigating the size of the microbubbles around single melanosomes by using fast flash photography scales proportionally with their lifetime according to the Rayleigh equation, which is also valid for cavitation bubbles. Nucleation temperatures have been experimentally found to be around $136{ }^{\circ} \mathrm{C}$ for ns [29] and $157{ }^{\circ} \mathrm{C}$ for $1.8 \mu \mathrm{s}$ pulse duration [27], respectively. These temperatures correspond to the nucleation temperature of water under a pressure of 3-4 bar, which also represents the surface tension of a microbubble that needs to be overcome for growing. Temperature and Arrhenius calculations using a mash of melanosomes interestingly also showed that the threshold dependence experimentally found with respect to the pulse duration is only applicable at the surface of the melanosomes [30].

$\mathrm{MBF}$ at melanosome clusters within RPE cells has been visualized by interferometry and fast flash photography, supported by vitality stains (Fig. 11.3) [31]. The dynamics of these bubble clusters reveal a nucleation on individual melanosomes and a later coalescence of these microbubbles to larger macrobubbles, especially with radiant exposures significantly above the threshold. Bubble wall speeds of typically $5 \mathrm{~m} / \mathrm{s}$ in the expansion phase were found, which reach up to $30 \mathrm{~m} / \mathrm{s}$ in the collapse phase [32]. In contrast to direct thermal expansion of tissue, the strong volume change due to bubble expansion is likely responsible for lifting the complete retina as observed in OCT [33]. When multiple microbubbles nucleate within a RPE cell, its volume increases instantaneously and disrupts the cellular membranes [34]. Consequently it is likely that this RPE damage mechanism can be attributed to a thermomechanical disruption.
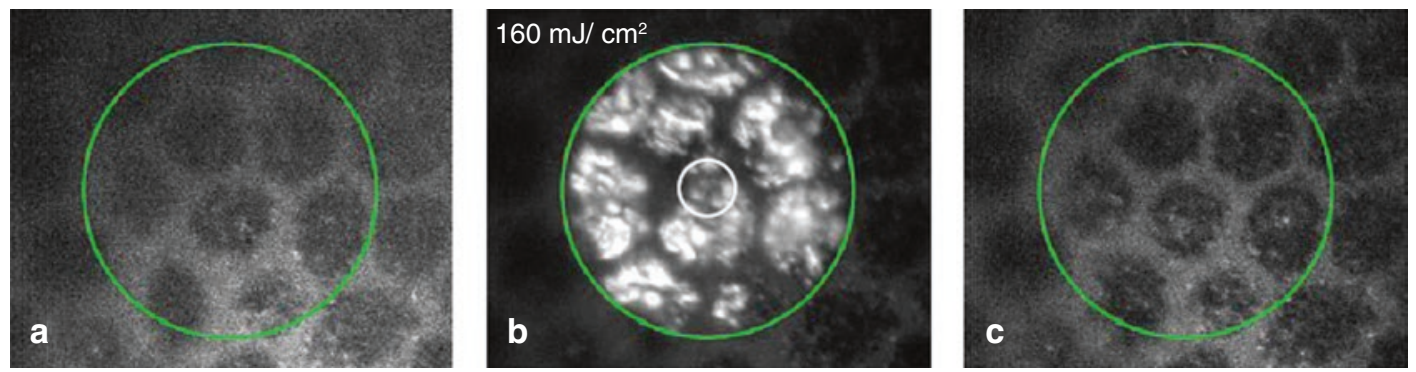

d

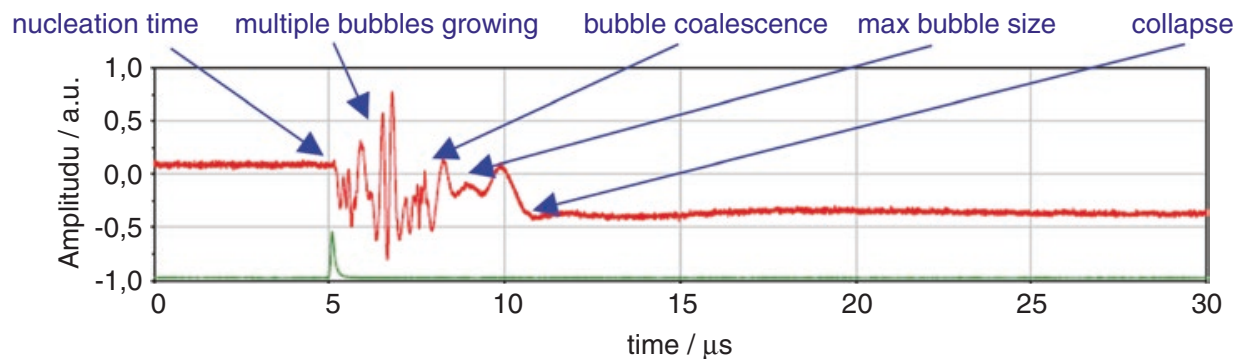

Fig. 11.3 RPE explant pictures [31] taken before (a), $200 \mathrm{~ns}$ after (b) and $1 \mathrm{~s}$ after (c) irradiation within the area (green circles). The highly reflective points (white regions) in (b) represent light scattered from multiple microbubbles. (d) Shows the laser pulse (green) and the interferometric transient (red) over time measured in the central circular area in (b) indicated with the white circle 
Experiments on rabbits in vivo revealed that RPE disruption is ophthalmoscopically invisible with white light illumination during treatment [35]. However, it can be demarcated by fluorescence angiography. In case of tight junction disruption within the RPE, fluorescein can diffuse through the broken blood retina barrier and fluorescing spots are observed at these locations. Typical $\mathrm{ED}_{50}$ threshold radiant exposures for $\mu$ s pulses (100 pulses with a single pulse duration of $1.7 \mu \mathrm{s}$ ) are determined by angiography to $131 \mathrm{~mJ} / \mathrm{cm}^{2}$, and therefore are comparable to those ones found on porcine RPE explants ex vivo labelled by a vitality stain. At radiant exposure about twice the $\mathrm{ED}_{50} \mathrm{RPE}$ damage threshold, effects become optical visible [12]. They appear like mild whitish photocoagulations, but can most likely be attributed to increased light scattering due to the mechanical dislocation and disruption of retinal tissue by large coalesced macrobubbles. With further increase of the radiant exposure, these macrobubbles lead to retinal/ choroidal disruption with bleedings as typically observed after Q-switched laser accidents. For a safe and selective treatment, a therapeutic window with radiant exposures slightly above the MBF threshold and far below twice the MBF threshold should be considered. When investigating the cellular damage mechanisms on pulse duration close to threshold irradiation in more detail, it shows that the predominant origin is thermomechanical for pulse durations up to $20 \mu$ s and thermal for pulse durations of $50 \mu$ s and larger $[36,37]$.

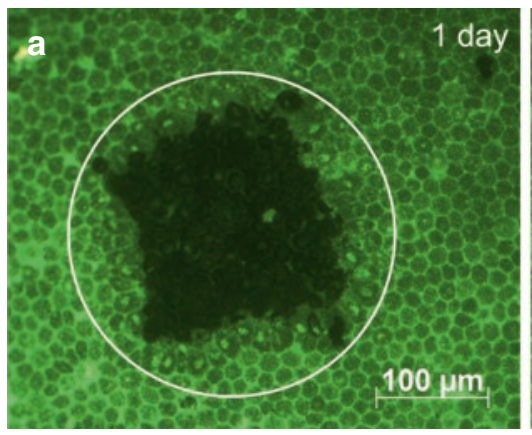

Fig. 11.4 RPE porcine explants irradiated with $140 \mathrm{~mJ} /$ $\mathrm{cm}^{2}$ per pulse stained with the vitality marker Calcein AM showing vital cells with green fluorescence [39]. Immediately after treatment the square laser beam profile
Histology and electron microscopy collected at different points in time after irradiation of rabbit eyes display disrupted RPE cells without damage of surrounding tissues [38], thus confirming the selectivity of RPE damage. Depending on the site, the wound is completely recovered with RPE cells within less than 1 week $[39,40]$ as shown in Fig. 11.4. As a first reaction, living RPE cells at the rim of the damaged zone extend and migrate into the ablation site and close it most rapidly. Further, cell mitosis and proliferation restore the original cell density (Fig. 11.5), which is considered to be the most important step towards regeneration of the RPE as the basic concept of the therapy. Histology further reveals that the photoreceptors in the adjacent layer remain functional. This is confirmed by the ongoing growing of their outer segments during wound healing due to the temporal absence of the RPE phagocytotic activity. Their normal length is resumed after wound healing is completed, which also proves RPE metabolic function. Multifocal electrograms of recovered tissue revealed no significant reduction in amplitude after SRT compared to standard photocoagulation as another proof of preserving retinal function [41, 42].

Cytokine release from RPE also alters after irradiation; an investigation with ex vivo RPE showed a decrease of the VEGF and an increase of the PEDF secretion from RPE cells 3 days after irradiation [39]. Proinflammatory cytokines, including IL- $1 \beta$ and TNF- $\alpha$, showed sig-

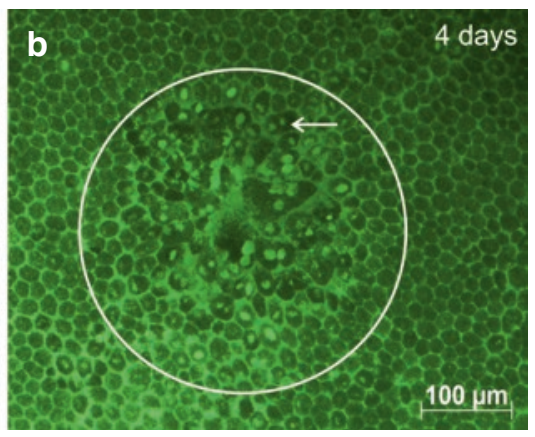

is visible (a). Four days after SRT, irregular shaped cells with conspicuous nuclei covered the laser-induced RPE wound (b). Some of these enlarged cells showed two nuclei (arrow), which suggest mitosis 


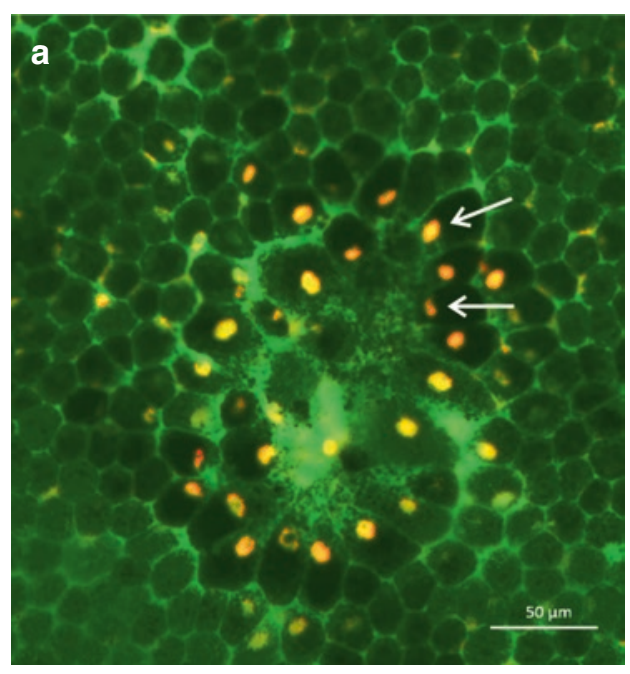

Fig. 11.5 Immunofluorescence staining of RPE-choroid explants [39]. (a) Displays mitosis indicated by the Ki-67 marker (orange) at days after SRT. Localized positive signals (arrows) (orange) in cells surrounding the SRT area indicate RPE regeneration. Untreated RPE showed no sig-

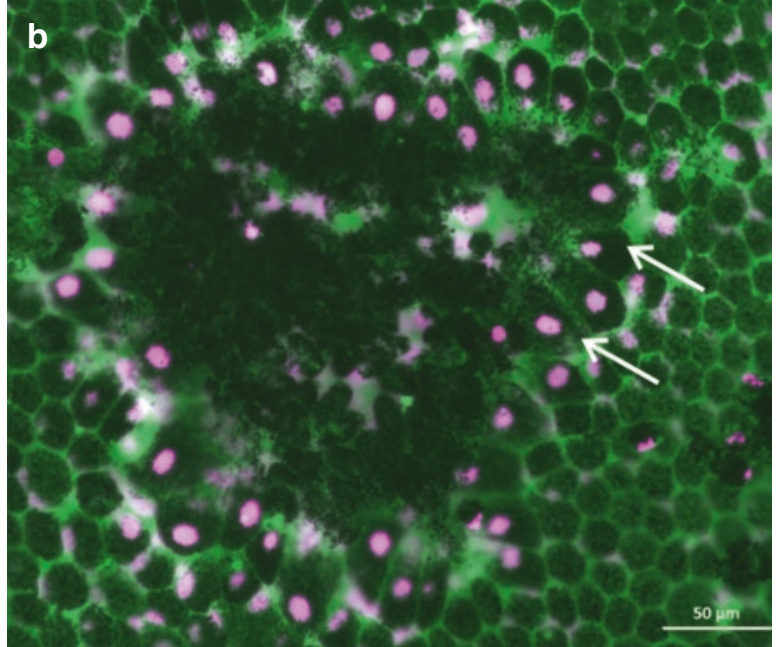

nal for Ki-67. (b) Using MMP-2 staining reveals a localized expression of MMP-2 (purple) in cell nuclei (b, arrows) of cells, which migrate into the SRT lesion after treatment, while untreated RPE only showed MMP-2 sporadically at cell membranes

\subsubsection{Clinical Study Results}

[43]. It has also been shown that matrix metalloproteinases (MMPs), particularly the active form of MMP-2 is significantly upregulated $[39,40,44]$ and also locally resolved as shown in Fig. 11.5 [39], which is assumed to play a positive role in preventing matrix degradation of Bruch's membrane, and eventually increasing transmembrane molecular exchange. Human donor samples of Bruch's membranes incubated with MMP-2 showed considerably improved hydraulic conductivity and transport capabilities $[17,40]$. Interestingly, the upregulation of active MMP-2 and PEDF in the whole RPE explant are more apparent with $200 \mu \mathrm{m}$ spots compared to $100 \mu \mathrm{m}$ spots when the total damage area was kept constant [39]. This suggests that the spot size of the RPE damage may also have an influence on the subsequent cytokine release and thus may affect the therapeutic effect. In conclusion, RPE cell regeneration together with an improved molecular diffusion rate through Bruch's membrane might contribute to the therapeutic effectiveness, as the complete choroidal-retinal complex is affected by SRT.
First SRT was undertaken to prove the concept clinically and to investigate its potential for treatment of different retinal diseases, focusing on three retinal pathologies: diabetic macular edema (DME), central serous retinopathy (CSR), and drusen in age-related macular degeneration (AMD). A Q-modulated frequency-doubled Nd:YLF-laser operating at a wavelength of $527 \mathrm{~nm}$ was used with a pulse duration of $1.7 \mu \mathrm{s}$ and a repetition rate of 100 or $500 \mathrm{~Hz}$ to apply 30 or 100 pulses, respectively, per treatment spot on the retina onto a diameter of $160 \mu \mathrm{m}[45,46]$. In order to find the sufficient pulse energies for successful irradiation closely above MBF threshold, titrations were performed prior to treatment at the arcades in combination with fluorescence angiography to demarcate the broken blood-retina barrier (Fig. 11.6). As observed in rabbits, SRT spots become directly optically visible by white light illumination at the slit lamp immediately after irradiation when exceeding a factor of about 2 above the angiographically determined threshold radiant exposure [47, 48]. 


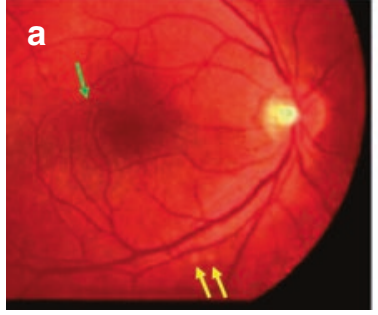

Fig. 11.6 Fundus picture (a) and fluorescein angiogram (b) of a CSR patient's fundus after SRT [47]. SRT lesions clearly show up in angiography. Test lesions with increasing energy for dosimetry were performed in the arcades, only highest pulse energies also lead to a slightly directly visible effect in the fundus photo (yellow arrows). Lowest angiographically visible radiant exposures were used for treatment in the central macula (green arrow). For comparison to standard photocoagulation, a fundus image after typical panretinal photocoagulation is shown in (c)
Lowest angiographically visible radiant exposures were found in the range of $350-500 \mathrm{~mJ} /$ $\mathrm{cm}^{2}$ per pulse in test expositions at the arcades, which is about 2.7-3.8 times higher than the rabbits angiographic $\mathrm{ED}_{50}$. For treatment, typically $650 \mathrm{~mJ} / \mathrm{cm}^{2}$ were used to compensate for the variation in pigmentation, which is typically lower in the central macula. It quickly turned out that the repetition rate of $500 \mathrm{~Hz}$ is too high for a spot diameter of $160 \mu \mathrm{m}$ in order to allow sufficient cooling between the pulses. Successively, until today $100 \mathrm{~Hz}$ is the standard repetition rate used.

The reason for the much higher required radiant exposures compared to the very young rabbit and pig is very likely related to the higher light scattering within the patient's eye before the radiation can reach the retina. Boettner and Wolter measured a strongly reduced direct light transmission through human eyes with age [49]. Only about $40 \%$ direct light transmittance in the green spectral range was measured for a 53 year old eye. This value further reduces with age owing to increased light scattering predominately in the lens due to age-related cataract, therefore well explaining the higher thresholds for clinical SRT. These findings also coincide with temperature measurements during retinal photocoagulation: Only about $20-30 \%$ of the green light entering the eye reached the target site at the retina and led to the temperature rise measured by optoacoustics in patients [50].

With respect to the therapeutic outcome in the follow-up period, hard exudates disappeared in 6 out of 9 patients, retinal edema resolved in 6 out of 12 diabetic patients, drusen were reduced in 7 out of 10 AMD patients and leakage in CSR disappeared in 3 out of 4 cases [46]. Investigations of SRT compared to photocoagulation lesions directly after treatment utilizing optical coherence tomography (OCT) revealed no OCT-visible hyperreflectivity at the SRT sites in contrast to the strongly scattering photocoagulation spots [51]. In both cases, the RPE appeared thinner in the follow-up. After 4 weeks, RPE thickening likely due to overproliferation was observed after SRT. One year after treatment, photocoagulation sites were characterized by RPE and neurosensory tissue atrophy, in contrast SRT lesions showed unaffected neurosensory structures and an intact RPE layer.

Encouraged by these promising pilot study results, an international SRT multicenter trial was conducted to evaluate the therapeutic effect of SRT in 60 patients with diabetic maculopathy [52]. In this study, 30 pulses with a single pulse duration of $1.7 \mu \mathrm{s}$ at $100 \mathrm{~Hz}$ were applied per spot with a top hat beam profile of $210 \mu \mathrm{m}$ on the retina. A $\mathrm{Nd}$ :YLF laser with intracavity overcoupled second harmonic generation was developed to generate $\mu \mathrm{s}$ laser pulses at $527 \mathrm{~nm}$ [53]. Typical pulse energies for DME treatments were 200-325 $\mu \mathrm{J}$, which refer to pulse powers between 117 and $191 \mathrm{~W}$ and calculated radiant exposures between 577 and $938 \mathrm{~mJ} / \mathrm{cm}^{2}$, respectively [52]. In $95 \%$ of the 60 patients the visual acuity improved or was stable at the 6 month follow-up control. The angiographic results with respect to leakage areas and OCT on edema size and thickness, however, didn't fully coincide with respect to the visual acuity. Further 
studies on DME [54], with macular edema after venous branch or central venous occlusion [55] and with geographic atrophy [56], were conducted.

Most impressive are study results for the treatment of central serous retinopathy (CSR). A study on 27 patients with active CSR showed a resolved subretinal fluid in $85.2 \%$ of the patients after 4 weeks and $100 \%$ after 3 months with no visible leakage. Mean visual acuity improved from $20 / 40$ at baseline to $20 / 20$ at 3 months [57]. A study randomizing patients to immediate treatment and 3 month waiting time also showed significant benefits and quick fluid dissolution for the patients treated immediately [58]. A study conducted in Japan supported these findings [59]. With respect to the safety of the treatment, microperimetry and multifocal ERG did not show any functional retinal defects, as typically found after standard photocoagulation [59]. Another pilot study conducted in Korea $(527 \mathrm{~nm}, 100 \mathrm{~Hz}, 30$ pulses per spot, $210 \mu \mathrm{m}$ diameter on retina) on chronic ( $>3$ months) CSR patients revealed much lower pulse energies between 65 and $90 \mu \mathrm{J}$ needed for selective RPE effects [48]. Angiographically visible effects were observed with as little as $70 \mu \mathrm{J}(200 \mathrm{~mJ} /$ $\mathrm{cm}^{2}$ ). Reasons for these lower radiant exposures are likely the relatively clear media of the younger CSR patients, the higher pigmentation of Asian eyes as well as the very strong intensity modulation on the lateral laser beam profile with that laser used. However, only 3.8 laser spots in average per patient were applied around the leakage point leading to a complete resolution of subretinal fluid in $75 \%$ of the patients after 9 months. Only very few spots around the feeder vessel need to be applied in the central macula, in contrast to a study using a non-damaging laser therapy using 548 laser spots in average per patient [60]. Thus for CSR, SRT seems to be an ideal treatment modality.

Another clinical study investigated shorter pulse durations between 200 and 300 ns for SRT, which also exhibited selective RPE damage and even lower pulse energies compared to $1.7 \mu$ s due to the reduced heat diffusion during irradiation $[25,61]$. However, since the individual nucleation times of the multiple microbubbles converge, the dynamics becomes more explosive and the risk of unintended bleedings rises, as clinically observed following the impact of single $3 \mathrm{~ns}$ laser pulses when using another technique for retinal rejuvenation [62].

The primary disadvantage during SRT treatment is the lack of direct optical feedback due to the low or missing visibility of SRT lesions. On the one hand, it strongly limits the ophthalmologist ability to interactively place the lesions in the desired areas. Neither reference grids for DME nor well localized circles or patterns in CSR treatment can be placed due to lack of visual feedback. On the other hand, with respect to proper energy dosage, studies showed a strong inter- and intraindividual variation of the necessary pulse energies for comparable RPE effects $[63,64]$. Even with titration at the arcades, angiography after treatment is required to ensure RPE defects due to the variability of the absorption. In case of negative angiography outcome, retreatment with higher energies is required, which is a tedious procedure with increased risk for the patient owing to multiple angiographies. The state of the art to improve and control dosage will be discussed in the next paragraph.

\subsubsection{Dosimetry and Dosing Control}

Microbubble formation (MBF) at the intracellular RPE melanosomes and their dynamics have been identified as the origin eventually leading to thermomechanical photodisruption of the cells $[22,34]$. As long as the irradiation takes place in the therapeutic window with radiant exposures slightly above the MBF threshold that only causes small separated microbubbles, a selective cell damage without any adverse effects to the photoreceptors and the choroid has been proven in multiple clinical trials $[45,46,48,51,52,54$, 55, 57-59, 61, 65-67]. MBF and blood-retina breakdown by the RPE disintegration shown by fluorescence angiography coincide very closely, hence the occurrence of microbubbles can be taken as a proof of successful RPE damage [63, 64]. Fortunately, microbubble formation and 
their dynamics can indirectly and non-invasively be measured in vivo during irradiation by different methods:
(a) Optoacoustics
(b) Light reflection
(c) Small bandwidth interferometry
(d) Broad bandwidth interferometry-OCT

With appropriately fast acquisition and rapid data evaluation, these techniques can be used to provide the attending physician with immediate feedback on the correct dosage. It can further be aggrandized for a fully automated feedbackcontrolled dosing to unburden the clinician from any manual dosing. As a useful strategy, the train of pulses per spot is ramped up and the occurrence of MBF is measured after each individual pulse. As soon as MBF appears the irradiation can be automatically ceased, either immediately or few pulses later if desired [68]. Figure 11.7a shows such a ramp with a stepwise increase over
15 pulses from 70 to $140 \mu \mathrm{J}$ exemplary. The technique has been approved in preclinical trials with automatic feedback control $[69,70]$.

With respect to clinical applications within the approval phase of such technique, currently the physician is guided by the system as follows: The clinician chooses the maximal energy of the last pulse and the system applies a stepwise ramp beginning with $50 \%$ of the maximal pulse energy. MBF is measured after each single pulse. After all 15 pulses have been applied, the system proposes to keep the energy for the next spot if MBF is measured with pulses in the middle of the ramp. In case of MBF at the beginning or end of the ramp, the physician is asked to decrease or increase the maximal energy, respectively $[54,71]$.

In order to detect MBF, optical and acoustical transients can be measured and evaluated with appropriate techniques for each individual pulse in real time, which will be explained in the following subsections. Exemplary Fig. 11.7 shows the applied energy ramp and a

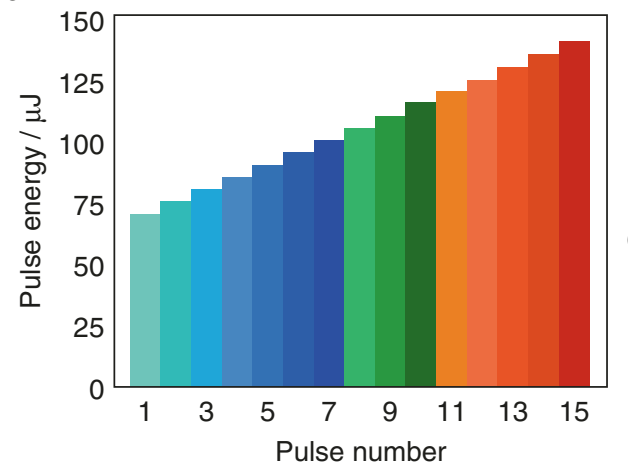

b

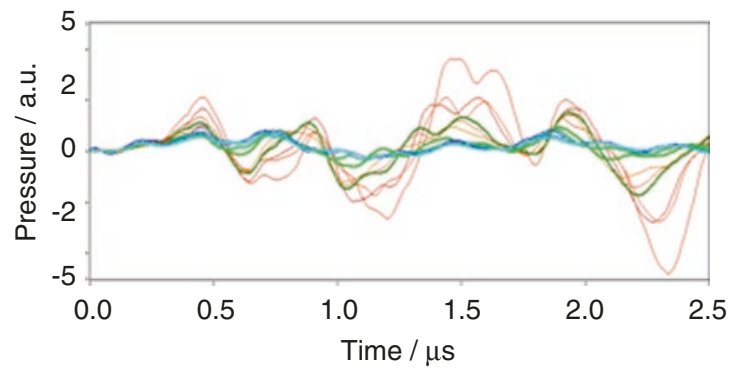

C

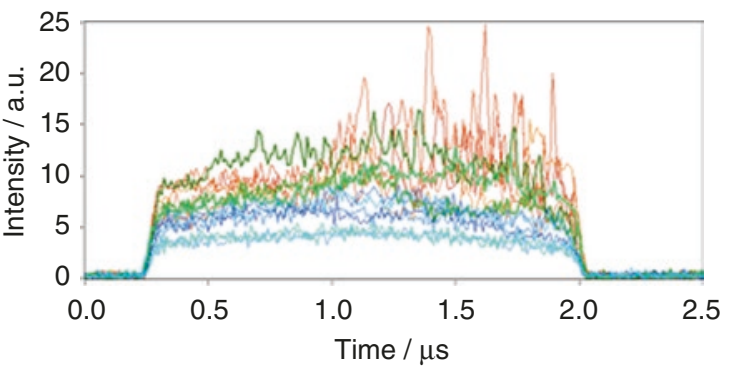

lines denote pulses with strong modulations associated with large and likely coalesced bubbles. In case of an automated feedback, laser emission should be ceased when reaching the green or orange pulses to safely stay within the therapeutic window in order to prevent large thermomechanical disruption. This can lead to collateral damage, including bleeding as it has been observed after therapeutic 3 ns laser exposure [62]
Fig. 11.7 Pulse energy ramp for a SRT spot (a) with acoustical (b) and optical transients (c) color-coded for the same laser spot, exemplary from a clinical trial using both techniques simultaneously [64]. Blue colours represent sub MBF energies. Pulse \# 8 (light green) first shows clear modulations on both the acoustical and optical transients, respectively, compared to the previous pulses, indicating that the MBF threshold has passed. Orange and red 
the according transients recorded during a clinical study at the University eye clinics in Kiel (UKSH) and Hannover (MHH) [64].

\section{(a) Optoacoustics}

Light absorbed by tissue is predominately converted to heat, which leads to its thermoelastic expansion and successive contraction by the emission of a bipolar pressure wave [72]. Its frequency depends on the thickness of the absorber and the spot size, while its amplitude is proportional to the pulse energy and the strongly temperature dependent Grüneisen parameter. Therefore optoacoustics can be used to calculate the tissue's temperature increase during SRT [73] and also during standard photocoagulation [50]. Typically, frequencies after heating tissue with ns to $\mu$ s pulses are in the $\mathrm{MHz}$ and therefore in the ultrasonic range. The acoustic waves travel back through the eye and can be measured at the cornea. As the pressure amplitudes are in the range of hundred microbars only, a very sensitive sensor is required. Typically, an annular shaped piezoelectric ultrasonic transducer is embedded in the contact lens, which is used anyway for laser treatments utilizing a laser slit lamp [73]. Figure $11.7 \mathrm{~b}$ (blue curves) show such transients when using SRT at low radiant exposures [64]. Since the pressure is proportional to the pulse energy, all transients are almost identical when normalizing on the energy (Fig. 11.7b). If the temperatures exceed the vaporization temperature at the surface of melanosomes, microbubbles form and expand $[74,75]$, and successively emit further individual pressure waves. As the nucleation onset times at the different melanosomes differ slightly, and also differ among different pulses owing to the displacement of the melanosomes by the bubbles, a phase and amplitude fluctuation of the transients is observed as exemplary shown in Fig. 11.7b (green and red curves). With an algorithm developed for taking into temporal deviations among the acoustic transients, Schüle et al. introduced an optoacoustic value (OA value) [63]. With an appropriate threshold OA-value, RPE damage upon fluorescein angiography can be predicted with high specificity [76, 77]. Also other algorithms based on further signal features can be processed, giving a higher security for the feedback when processed in parallel [77]. An appropriate threshold OA-value can be used in real time to interrupt the laser ramping and emission after MBF exceedance. The beauty of this approach is that the excitation is induced by light while the response is based on acoustics, since the pressure waves are almost not affected by the ocular media. From the perspective of bubble formation, the correct focus of the light or the beam profile itself play only very minor roles. The technique has been proven in clinical studies by displaying the OA-value to the ophthalmologist via a monitor $[52,63]$ or verbally [59] in order to give them immediate feedback whether the irradiation energy was sufficient. In recent clinical studies, it was also used for dosing guidance [54, 71].

(b) Light reflection

During laser irradiation of the retina, some of the treatment light is scattered back from the RPE and can be measured in front of the eye. If only tissue heating takes place, its reflective properties stay almost constant and the pulse shape of the back-reflected light is identical to the incoming one (Fig. 11.7c). After MBF however, microbubbles cause a refractive index change at the bubble's surface between tissue on the outside, essentially consisting of liquid water $(n=1.4)$ and water vapor inside the bubble. $(n=1)$ of about 2-3 orders of magnitude. Further the light is modulated owing to the dynamics of the bubble expansion and collapse. Figure 11.7c (green and orange curves) shows such back-reflected pulse shapes which strongly deviate from those below bubble-formation threshold. This method was developed by Seifert et al. [70] with respect to calculate a reflectometry value (RE-value). Data from clinical trials were recently analyzed and mathematically optimized in order to obtain a threshold RE-value with highest sensitivity and specificity [64] for an automatic real-time stop of the pulse energy ramp. The technique is currently under investigation in clinical trials 
$[54,64,71,77]$. The strength of this method is that the high power laser light used for treatment can be evaluated directly. Therefore on the hardware side, nothing but a fast photodiode is required to record the strong backscattered light. However, in contrast to optoacoustics, the reflected light intensity is influenced by the ocular media as lens and cornea and can strongly vary as also the backscatter of a range of small spherical 'bubble-foam' is highly dependent on the particular microbubble distribution.

(c) Small spectral bandwidth interferometry

The occurrence and dynamics of microbubbles can be well probed by interferometry using small bandwidth laser light with large coherence length as typically provided by single longitudinal mode lasers. By means of a fast photodiode collecting the reflected light from the eye in the object arm of the interferometer, microbubble dynamics can be recorded at very high temporal resolution and thus provide more information than just the MBF threshold detected by OA- and REvalues. With a single frequency laser diode, the heating of the tissue in form of a slight intensity shift due to the thermal expansion of the RPE, as well as the onset of microbubbles (modulations in Fig. 11.3), their coalescence and collapse, and finally the slow thermal contraction after irradiation have been monitored $[31,32]$. Thus small bandwidth interferometry can likely be used for fine dosing control in SRT [78], however has not been developed far enough for approved clinical trials.

(d) Broad spectral bandwidth-OCT

Low coherence or white light interferometry (LCI) responds similarly to reflectivity changes like any of the other optical approaches. The broad bandwidth and the coherence between the different wavelengths, however, can also be utilized to transmit spatial information of the reflector in the light propagation direction. Optical coherence tomography combines this technology with a lateral scan and enables to obtain images of reflectivity in form of axiolateral cross-sections or volumes. The avail- ability of performing OCT during retinal therapy exposure for observing signal variations correlated to the creation of RPE lesions has already been investigated at times where early frequency-domain OCT system have become widely available [79]. However, at that time the temporal resolution was only sufficient to monitor thermal expansion of tissue. For real-time diagnostics of laser-tissue interaction, two time-frames that require quite different acquisition speeds have to be distinguished. One is the thermal expansion regime that is specified by thermal diffusivity in the range of up to $0.16 \mathrm{~mm}^{2} / \mathrm{s}$ for biological tissue [80], leading to speeds of $\sim 80 \mathrm{~mm} / \mathrm{s}$ and the other is where acoustic waves with $\sim 1500 \mathrm{~m} / \mathrm{s}$ dominate the energy transfer to surrounding tissue [81]. Several groups have investigated structural OCT as an imaging modality to compare retinal layers before and after treatment [51, 82], or functional Doppler OCT for thermal expansion measurements [79, 83]. This direct imaging of thermal expansion during the sufficiently slow heat diffusion process is straight forward with current OCT systems at moderate axial resolutions $\sim 10 \mu \mathrm{m}$ and $\sim 100 \mathrm{kHz}$ depth scan rates even for the lateral crosssections of OCT tomograms. Although modern OCT systems can scan remarkably faster ( $>1 \mathrm{MHz}$ depth scan rate, being $<1 \mu$ s exposure time per depth scan) along with a reduction of temporal resolution of the lateral scan, the 4 orders of magnitude faster shockwaves induced by a short treatment pulse in the sub $10 \mu \mathrm{s}$ scale cannot be directly resolved in an 2D image. This becomes even more restricting in typically slower ultrahigh-resolution systems with a spectral bandwidth of more than $100 \mathrm{~nm}$ that supports an in vivo axial resolution of about $3 \mu \mathrm{m}$ or below, which is required for clear visualization of cellular structures in the RPE layer and differentiation from its surrounding tissue [84].

During a burst of successive pulses, optical reflectance from the irradiation laser increases in signal strength. Feedback from reflectance of 
a forming microbubble is a simple optical technique for thresholding the exposure [41], as has already pointed out above. LCI, or single position, non-scanning OCT utilizes the full temporal bandwidth for real-time SRT quantification of the shockwave formation and can monitor effects caused by shockwaves even at $\mathrm{kHz}$ scan rates. In OCT systems it is thus an elegant method to reuse an existing detection channel even though it sacrifices the lateral scan directions. Such timeresolved OCT has been utilized to detect slower effects in even less aggressive retina-light interaction in so called optophysiology before it was implemented for SRT [33, 85]. Large distortions induced by laser pulses can be indirectly detected as a temporal change of intensity in OCT depth scans (A-scans, amplitude mode), which corresponds to the local reflectivity of tissue. An M-scan (motion mode) visualization, which is a time-resolved sequence of A-scans facilitates the recognition of differences (Fig. 11.8b). To extract changes in the structure time frequency analysis (TFA) of the signal can help to detect subtle changes in the M-scan. Shorter spikes only covering part of the frequency spectrum indicate slower temporal fluctuations within the M-scan (red vertical spikes in Fig. 11.8c at start and end of the laser irradiation are indicated by arrows). Large interruptions become apparent as stripes across all frequencies. In the M-scan zoom-in (Fig. 11.8a), these changes are obvious blackouts with complete signal deletions.

\subsection{OCT for SRT Dosimetry}

\subsubsection{Hypothesis of Fringe Washouts in M-Scan OCT}

The origin of the above-mentioned complete OCT signal loss can be explained by coherent signal washout. The coherent decorrelation or "fringe-washout" results from rapid fluctuations of the spectral phase, which are faster than the integration time of the detector, such that the time-averaged signal becomes almost zero. This effect is well known in OCT images of larger blood vessels, especially for those oriented parallel to the measurement beam. Their lumen appears dark due to the fast turbulent blood flow of moving cells that generate fast modulation of the phase [87]. This effect differs from intensity
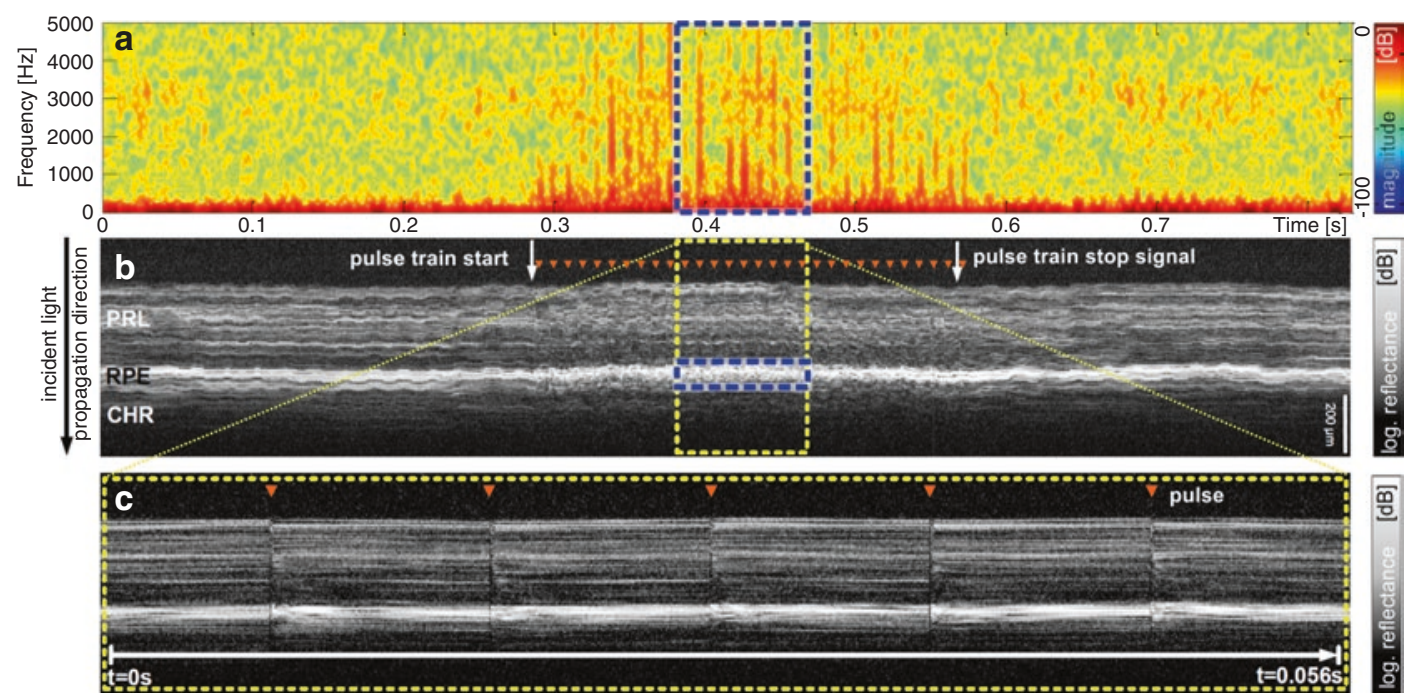

Fig. 11.8 OCT M-scans of an ophthalmoscopically invisible SRT treatment lesion on ex vivo porcine eyes at high pulse energy and radiant exposure, well above the threshold for microbubble formation $(180 \mu \mathrm{J}, 570 \mathrm{~mJ} /$ $\mathrm{cm}^{2}$ ) [86]. (a) Time-frequency analysis (TFA) of speckle pattern at the RPE (blue-white dashed box in $\mathbf{b}$ ) as well as an extract of $56 \mathrm{~ms}$ during laser application (c, yellow dotted box) clearly visualize changes caused by SRT application in both M-scan and TFA data (red arrowheads indicate pulse positions) 
decorrelation, where the variation between successive OCT scans is numerically calculated for contrasting motion, while in coherent decorrelation the OCT signal's phase is already destroyed prior digitization. In coherent or phase decorrelation, the tissue scatterers are moved by a fast oscillating sound field at amplitudes in the range of the axial resolution, therefore the spectral phase is altered quicker than the exposure time of the irradiation pulses.

A typical OCT system operating at 20-100 kHz acquisition speed cannot resolve motion above $100 \mathrm{~mm} / \mathrm{s}$ or equivalent speckle fluctuations and therefore will start to experience signal losses when the cohesion of neighboured scatterers within the OCT resolution volume is lost, which corresponds well to microbubble sizes of several micrometres. This distinct attenu- ation can be utilized as a sensitive indicator for the occurrence of strong axial motion associated with laser-induced microbubble formation within the monitored volume. Closer investigation of the M-scans reveals also smaller signal fluctuations that correspond to the smaller spikes in the TF analysis (Fig. 11.9d). In the M-scan these distortions exhibit a logarithmic decay that fits thermal dissipation of heat as expected by previous simulations [22].

This ability to discriminate effects due to sole heat-related changes from microbubble-related ones is a viable advantage to simple reflectometry. The reflectometry utilizes a swelling signal that is supposed to correlate with vapour-bubble size [89]. In OCT, however, no growing of signal intensity is found throughout the M-scan within a burst sequence, which indicates that decorre-
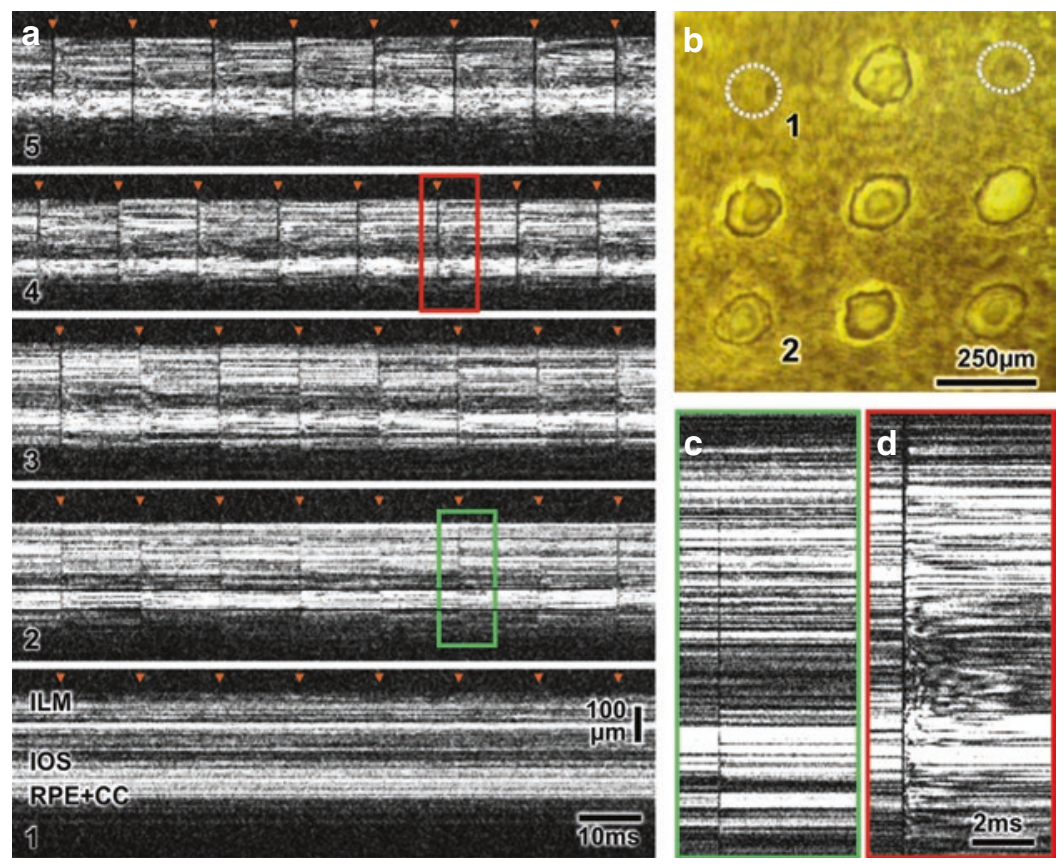

Fig. 11.9 (a) M-scans of ex vivo treatments at multiple locations with constant pulse energy at $1.7 \mu$ s pulse length. Pulse energy increases from M-scan number 1 to 5 from 40 to $200 \mu \mathrm{J}$ (fluence: $200-1000 \mathrm{~mJ} / \mathrm{cm}^{2}$ ). In the lowest energy M-scan no apparent signal washout is visible, while it increases with irradiance. (b) Top view onto RPE layer under bright field illumination. Strong lesions (5), with complete annihilation of the RPE relate to strong signal washouts in the corresponding M-scan. Weak lesions (2) conversely relate to weak M-scan signal washouts.
Spots without visible lesions in the RPE layer (1) correlate to M-scans without any signal washout. Magnifications of the signal washouts (vertical black lines) in M-scans induced by (c) a single laser pulse with $54 \mu \mathrm{J}$ and (d) a single laser pulse with pulse energy of $180 \mu \mathrm{J}$ indicate a difference in length of the signal loss and furthermore thermal distortions/fluctuations of the speckle for a longer time $(\sim 4 \mathrm{~ms})$, especially in the retinal center, above the $\mathrm{RPE} / \mathrm{CC}$ complex. Adapted from [88] 
lation superposes the increase in reflectivity and can act as a sensitive indicator for bubble buildup. The distortions introduced are not completely independent of the bubble size; although there seems to be a slight upward drift within and between successive scans of the inner limiting membrane (ILM) signal edge that might indicate a vertical shift due to bubble formation within a burst-sequence; this also could be contributed to thermal expansion and is hard to interpret due to the speckle. In contrast, the length of the blackouts correlate well with radiant exposure/fluence and stronger pulses also experience a stronger structural change as well as an elongated relaxation period (Fig. 11.9).

\subsubsection{First Pre-clinical and Clinical Studies}

Pre-clinical studies could show that the signal changes in OCT M-scans during SRT allow real-time prediction of retinal lesions $[33,86$, $88,90,91]$ also found in clinical experiments. Figure 11.10 demonstrates sub-visual (no visible coagulation spot in the fundus image e) in vivo treatment with clear response in the OCT scan in addition to a brightening in the fundus fluorescence angiography (FFA) scan after application of a 30 times pulse burst sequence at $\sim 160 \mathrm{~mJ} /$ $\mathrm{cm}^{2}$ fluence per pulse applied at $100 \mathrm{~Hz}$ repetition rate and $250 \mathrm{~ns}$ pulse length. This complies with the exposure levels for microbubble formation from previous studies, where the calculated fluence for MBF is expected between 140 and $240 \mathrm{~mJ} / \mathrm{cm}^{2}$. The calculated cumulative heating below $2{ }^{\circ} \mathrm{C}$ is contrary to data from quasi-cw exposure where the threshold for visible lesions was found at a ten times larger local temperature increase [79]. The absence of such a significant temperature increase opens a large laser process/ treatment window, before neighboured tissue is affected.

\subsubsection{Future Developments Towards Reliably Detecting the Microbubble Threshold with OCT}

Several strategies for limiting the exposure beneath the threshold for retinal heat damage have been discussed and exploited. Simply keeping the average power well beneath the average levels for coagulation misses the therapeutic window that spans between pure RPE and extended retinal damage due to the strong local absorption variations of about an order of magnitude. With typical average excursions of $\sim 0.5^{\circ}$ during microsaccades that mostly stay smaller than $1^{\circ}$
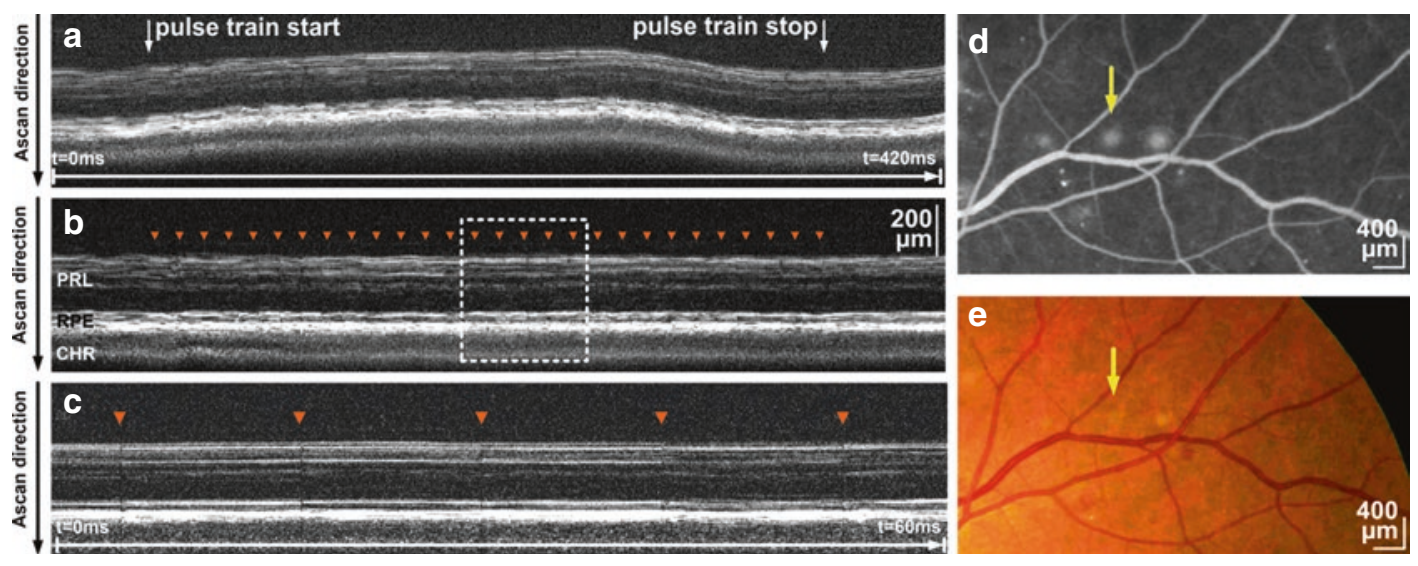

Fig. 11.10 OCT M-scan of successful in vivo SRT treatment (a) with axial position tracking (b). (c) Represents the inset in (b, dotted box) with white arrows indicating SRT pulse application with $80 \mu \mathrm{J}\left(160 \mathrm{~mJ} / \mathrm{cm}^{2}\right.$ at $\left.250 \mathrm{~ns}\right)$. (d) and (e) show the corresponding FFA and the fundus image. Yellow arrows correspond to the position of the treatment spot shown in the OCT scans. Adapted from [86] 
corresponding to shifts of $\sim 288 \mu \mathrm{m}$ on the retina during fixation focussing of treatment spots to $\sim 200 \mu \mathrm{m}$ is challenging. Therefore, repetitive application of short pulse bursts between microsaccades ( $\sim 200 \mathrm{~ms})$ to shorten the time frame below the instability of the human eye during fixation, is one way to adapt to the situation as it was suggested by the reflectometry controlled SRT model [89, 92].

Under the presumption that the appearance of a microbubble already indicates irreversible damage to the RPE and OCT reliably relates detected shockwaves to microbubble appearance, another approach is more sensible. Here the pulse train is successively ramped up in fluence until a MBF signal is reached or the maximum permitted exposure at full absorption is reached (see also Fig. 11.7a).

To successfully utilize a ramp mode, adaptive laser pulse energy control together with a fast and reliable method for exposure interruption is required. On top of the signal washout, which can be detected by simple signal thresholding the high sensitivity of OCT carries more information hidden in the sub-structure of the M-scan, including thermally induced fluctuations and possible secondary changes due to the microbubble formation. One way is to use human interpretation of the signals and to train a synthetic classification network. The network successively learns how to interpret different preselected signal features such as artifacts that compare subblocks with and without laser exposure, speckle changes, changes in the speckle variance or spectrum, similar to the TF analysis mentioned above and associates their appearance to the outcome. Besides the teaching with less clear in vivo data and direct human observation of the OCT signals together with TF analysis, this network can also be trained with ex vivo data that is based on histological results to objectively relate to selective RPE damage. As assumed, features found within the first $100 \mathrm{~ms}$ after exposure deliver the highest yield, while later portions of the signal between 100 and $300 \mathrm{~ms}$ after the exposure pulse continuously lose their relation to the distortion. Such automated analysis boosts prediction to success rates from initial $\sim 60 \%$ beyond $90 \%$ when targeting for $95 \%$ specificity [93].
Artificial convolutional neuronal networks can take this approach even a step further to select their own internal features, rather than predefined ones. This currently en vogue industry driven approach has the advantage of exploring and utilizing information that has not been considered by the human supervisor, but also bears the risk to train features that are not directly related in a cause-effect relationship that is further encoded in deeper hidden network nodes. A typical example is an artificial neuronal network that is trained to distinguish dogs from wolves, but in the end only learns to react on the snowy background most frequently found in wolf pictures. Similar risks are found in the SRT application where patient, image background signals or system properties can influence the results, unless very large numbers of verified examples are used. The training of such a network on OCT signals, however, also brings novel insights that are significantly harder to quantify by a human observer. When investigating the significance of signal components during the time course for predicting the accuracy of the treatment outcome, it can be shown that window sizes of $\sim 4 \mathrm{~ms}$ which also contain information from the status $200 \mu$ s prior to the applied pulse for the utilized system achieve the highest precision and recall, or in other words the lowest false positive and false negative rate [94]. An explanation for this behaviour is that $200 \mu \mathrm{s}$ before exposure, relatively slow changes due to eye-motion do not affect the baseline, while longer ones already exhibit distortions.

For integration into a clinical system these artificial neuronal networks have the advantage of shifting the complexity into a hidden layer that requires enough high-quality data for training, but thereafter can be realized with hardware that is capable to perform decisions in real time even with these sophisticated tasks. Specialized hardware with low energy consumption, high efficacy and speed is currently becoming available. Nevertheless, a black box system for application in laser therapy implies strict control mechanisms. Assuming 5-30 steps of treatment pulses with increasing energy within the stable exposure window of $200 \mathrm{~ms}$, reaction times of $\sim 3-20 \mathrm{~ms}$ are the target for ramp interruption. 
Considering electronic acquisition and laser driver delays, such a decision has to be made within a millisecond or has to be performed one or several pulses later when the pulse energy has already grown. Optimizing the ramps start, end levels and slope will be a question to be answered together with the choice of the definite detection mechanism.

The following chapter goes back from this outlook to the current technical efforts and improvements. It describes in detail the realization of a complete prototype setup combining OCT-based dosing control, therapy planning and documentation.

\subsection{SRT Module Integration into the OCT Platform}

Early-on the Heidelberg SPECTRALIS platform, originally built on a confocal scanning ophthalmoscope already hosted a robust spectrometer-based frequency-domain OCT system and was capable of tracking lateral eye motion, circumventing the above-mentioned issues by establishing a different approach: Here the eye tracker allows for sufficiently precise and fast online tracking of the scanners lateral position such that imaging of OCT cross-sections become re-entrant. This means that scans can be interrupted and restarted at any point during the scan, and that imaging sessions can be repeated in long-term studies as "follow-ups", since the tracking algorithm aligns the optics with the long-term stable retinal vasculature, even with poor quality ocular media. Furthermore, the modular design of this commercial multimodal platform also provides access to other wavelength ranges, which can equally benefit from the eye tracker, as well as the integration of other optical measurement or treatment devices.

In a first collaboration with Heidelberg Engineering, the optoLab of the Bern University of Applied Sciences in Biel has modified the mechanical setup of the SPECTRALIS platform and has successfully integrated a second OCT spectrum-scanning light source at $1050 \mathrm{~nm}$ into the system. With this setup named SPECTRALIS Hydra, a simultaneous acquisition of OCT images in both wavelength ranges became possible. First clinical studies at highly myopic children have been performed in Hong Kong to examine early aged myopia [95].

Learnings from this implementation including the mechanical setup and electronic communication enhancements were the basis for integrating a retinal treatment laser instead of a second OCT light source. This new experimental setup named SPECTRALIS Centaurus offers a fast, smart and minimally invasive clinical tool for performing SRT.

The SPECTRALIS Centaurus system integrates hard-, firm- and software to deliver OCT controlled, automatically tissue-adapted power and exposure-limited pulses with the high lateral and axial accuracy provided by the confocal eye tracker [96]. The $532 \mathrm{~nm}$ treatment laser is collinearly coupled into the light path of the $870 \mathrm{~nm}$ broadband OCT spectrometer via a dichroic beam splitter after adjusting the spot diameter. Furthermore, the beam from the cSLO centred at $820 \mathrm{~nm}$ overlaps collinearly on the return path from the eye. To enable other imaging modalities such as $486 \mathrm{~nm}$ fluorescein angiography and $786 \mathrm{~nm}$ ICG (indocyanine green) angiography, the corresponding lasers are also integrated (see Fig. 11.11).

The SPECTRALIS Centaurus system features a novel compact $\mu$ s laser system built on the experience with its predecessors. The experimental MERILAS SRT laser (Meridian AG) operates at the upper pulse length limit for SRT in comparison to its predecessors (Table 11.1). Not implementing a Q-switched laser that can accumulate energy is a choice that limits available fluence to shorter exposure times, but considerably eases implementation. With a spot diameter of $120 \mu \mathrm{m}$ that can be reduced due to the ocular stabilization, the heat losses are slightly stronger than with $200 \mu \mathrm{m}$ spots, but the radiant exposure is considerably increased. With a maximum radiant exposure just above the upper microbubble threshold, the linear growth of energy with pulse length is faster than the increase of the threshold and achieves about $150 \%$ at $4 \mu$ s correspond- 


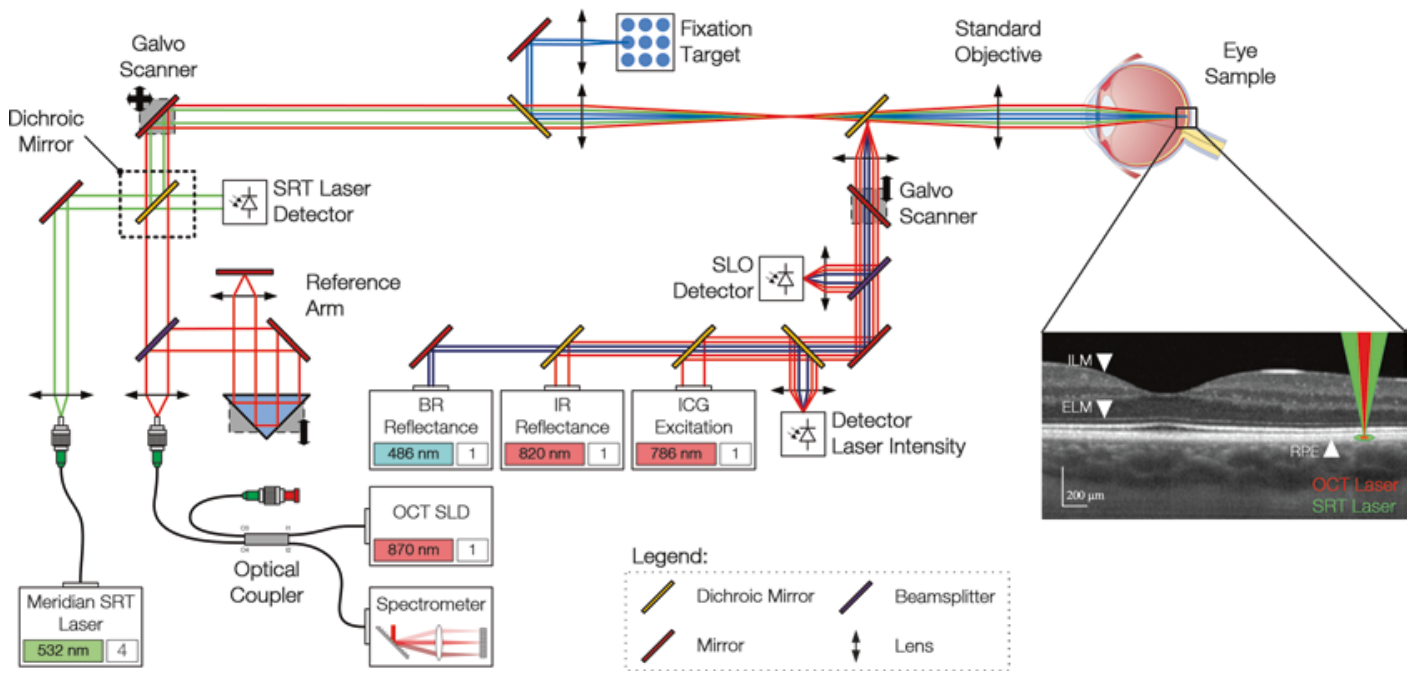

Fig. 11.11 Configuration of the SPECTRALIS Centaurus system [96]. The $532 \mathrm{~nm}$ treatment laser is integrated into the specially modified optics of the adapted
OCT system (left portion in front of the galvanometric scanner pair) and allows interaction with the multiwavelength laser tracking and exposure system

Table 11.1 Overview of parameters used for different SRT investigation systems

\begin{tabular}{l|l|l|l|l|l}
\hline & & Steiner 2014 & Steiner 2015 & Kaufmann 2018 & Burri 2018 \\
\hline Tissue & & Porcine ex vivo & Human in vivo & Porcine ex vivo & Porcine ex vivo \\
\hline Wavelength & $\mathrm{nm}$ & 577 & 527 & 527 & 532 \\
\hline Spot diameter & $\mu \mathrm{m}$ & 130 & 170 & 160 & $100-120$ \\
\hline Pulse duration & $\mu \mathrm{s}$ & $10-33$ & $0.2-0.25$ & 1.7 & $2-20$ \\
\hline Pulse energy & $\mathrm{mJ}$ & $0.7-20$ & $0.04-0.14$ & $0.013-0.34$ & $0.01-0.42$ \\
\hline Burst length & $\mathrm{ms}$ & 10 & 7.5 & $10-300$ & $2-300$ \\
\hline Repetition rate & $\mathrm{Hz}$ & 100 & 100 & 100 & $100-500$ \\
\hline OCT scan speed & $\mathrm{kHz}$ & 60 & 70 & 50 & 40 \\
\hline Axial resolution & $\mu \mathrm{m}$ & 1.3 & 1.3 & 1.8 & 7
\end{tabular}

ing to $\sim 350 \%$ of the minimum threshold fluence measured in melanosome heating experiments [22]. It will have to be seen if in clinical practice with patients with increased ocular opacity, i.e. due to cataract, these levels are sufficient for all treatment cases or higher laser pulse energy could be required. Anyhow, spot stabilization due to the ability of real-time eye tracking allows for even smaller spot sizes at the retina, with the side effect of more precisely mapped treatment areas. Furthermore, the system provides an easy way to perform standard photocoagulation treatment with the same system by selecting longer pulses.

Electrical signalling and firmware are already integrated together with basic software that might be extended for planning automated treatment purposes, such as automated mapping of a predefined region based on the fundus scan. In anticipation of clinical in vivo imaging and treatment the SPECTRALIS Centaurus system was adapted to support mounting of enucleated eyes (Fig. 11.12). Preliminary measurements already show results comparable to the preceding studies.

With first encouraging ex vivo experiments performed at the optoLab and the Medical Laser Center Lübeck (MLL) as well as the technical safety evaluation, it is planned to validate the SPECTRALIS Centaurus system for clinical in vivo studies. 

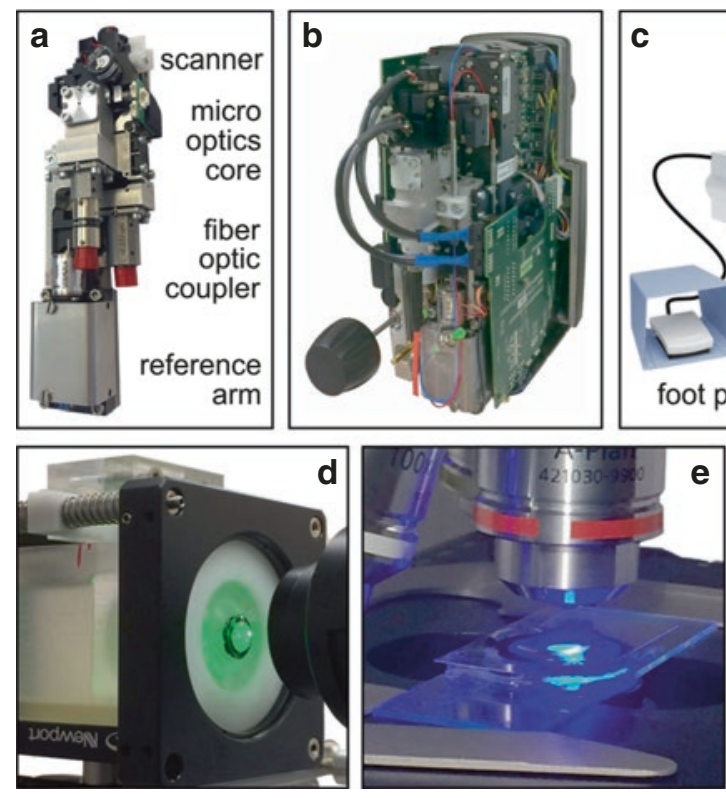

Fig. 11.12 Overview of the SRT-OCT setup and the measurement protocol [96]. (a) The SRT upgrade module has become an integral part of the SPECTRALIS platform and is (b) inserted into the compact measurement head. (c) The treatment laser is controlled by a foot switch and can be optionally suppressed by the real-time OCT safety

\subsection{Conclusions and Outlook}

The SPECTRALIS Centaurus system integrates a $\mu$ s pulsed treatment with a high-speed confocal laser scanner in a compact design. Despite not being a commercial device, the platform has shown a possible way to clinical implementation. In addition to the long-term stable optomechatronic design, the "commercial-level class" platform features high reliability, low maintenance, combined with a simple and userfriendly interface and remote maintenance of the device. The integration of a fast, high-resolution 3D imaging modality with therapeutic capabilities enables detailed planning of precise control during potentially harmful treatment. The exact positioning of the treatment laser pulse in time and space, independent of the patient's movement, as well as the possibility of immediate pre-/post-diagnosis, and a feedback system allows for an unrivalled accuracy and fulfilment of the physician's pre-set targets including safety features that avoid overexposure of the retina. Current approaches for limiting exposure are already well developed and have started to become indispensable tools for minimally invasive optical surgery. The ophthalmic instrument market has shown that contactless, all-optical technologies have always been favoured by clinicians and patients alike, due to their simpler, speedier and safer application with higher convenience and lower risk of mechanical injury or of cross-contamination. Even though the principle mechanisms of laser tissue interaction are well known, the variability of biological materials and delicate alignment of the treatment system demand for considerably increased control for each individual exposure. Precision-guided in vitro experiments already suffer from variations caused by inhomogeneity of melanosome density and absorption coefficients within the RPE layer. To transfer the results of ex vivo trials to clinical ones that add multiple layers of complexity and variation, a high reproducibility 
of the instrument during clinical application is required. Literally in focus of the research, the critical laser parameters are linked to the available technology and reveal only parts of the theoretical possibilities. As novel compact and cost-effective lasers open up new possibilities, the perfect choice of laser treatment for certain diseases will require more advanced studies that will definitely benefit from a multi-functional device that provides full control during the application of the parameters, along with an advanced diagnostic toolset for planning, performing and reviewing or even aftercare. This not only provides the surgeon with a more precise instrument that improves the statistics, but also allows the patient to benefit from an optimal and individual treatment that results in a therapeutic outcome which close resembles the original plan and mitigates risks and unwanted side effects.

Acknowledgments We gratefully want to acknowledge the continuous and valuable support from the team members from Bern University of Applied Sciences in Biel: Christian Burri, Michael Peyer, Patrick Steiner, Daniel Kaufmann, Mathias Mooser, Patrik Arnold, Tiziano Ronchetti, Volker M. Koch, Joern Justiz, Anke Bossen, Patrick Morgenthaler, Dominik Inniger, Christoph Meier, the Medical Laser Center Luebeck: Dirk Theisen-Kunde, Veit Danicke, Alessa Hutfilz, the colleagues from Meridian AG, Thun: Michael Stetter, Rudolf von Niederhäusern, Eric Odenheimer and the Heidelberg Engineering team: Stefan Schmidt, Michael Reutter, Joerg Fischer, Tilman Otto that enabled the development and implementation of this technology.

\section{References}

1. Meyer-Schwickerath G. Lichtkoagulation; eine Methode zur Behandlung und Verhütung der Netzhautablösung. Albrecht Von Graefes Arch Ophthalmol. 1954;156(1):2-34.

2. L'Esperance F Jr. Clinical applications of argon laser photocoagulation. Trans Ophthalmol Soc U K. 1970;89:557-73.

3. Birngruber R, Hillenkamp F, Gabel VP. Theoretical investigations of laser thermal retinal injury. Health Phys. 1985;48(6):781-96.

4. Early Treatment Diabetic Retinopathy Study Research Group. Early photocoagulation for diabetic retinopathy: ETDRS report number 9. Ophthalmology. 1991;98(5):766-85.
5. Early Treatment Diabetic Retinopathy Study Research Group. Photocoagulation for diabetic macular edema. Arch Ophthalmol. 1985;103:1796-806.

6. The Macular Photocoagulation Study Group. Agerelated macular degeneration. Am J Ophthalmol. 1984;98(3):376-7.

7. Nicholson B, et al. Central serous chorioretinopathy: update on pathophysiology and treatment. Surv Ophthalmol. 2013;58(2):103-26.

8. Findlay Q, et al. Prophylactic laser in age-related macular degeneration: the past, the present and the future. Eye. 2018;32:972-80.

9. Park YG, Kim EY, Roh YJ. Laser-based strategies to treat diabetic macular edema: history and new promising therapies. J Ophthalmol. 2014;2014:769213.

10. Marshall J. Thermal and mechanical mechanisms in laser damage to the retina. Invest Ophthalmol Vis Sci. 1970;9(2):97-115.

11. Kim HD, et al. Retinal pigment epithelium responses to selective retina therapy in mouse eyes. Invest Ophthalmol Vis Sci. 2016;57(7):3486-95.

12. Framme $\mathrm{C}$, et al. Influence of pulse duration and pulse number in selective RPE laser treatment. Lasers Surg Med. 2004;34(3):206-15.

13. Kozak I, Luttrull JK. Modern retinal laser therapy. Saudi J Ophthalmol. 2015;29(2):137-46.

14. Marmor MF, Wolfensberger TJ. The retinal pigment epithelium. Oxford: Oxford University Press; 1998.

15. Gabel VP, Birngruber R, Hillenkamp F. Visible and near infrared light absorption in pigment epithelium and choroid. Congress Series: XXIII Concilium Ophthalmologicum. 1978;450:658-62.

16. Schraermeyer U, Heimann K. Current understanding on the role of retinal pigment epithelium and its pigmentation. Pigment Cell Res. 1999;12:219-36.

17. Ahir A, et al. Expression of metalloproteinases from human retinal pigment epithelial cells and their effects on the hydraulic conductivity of Bruch's membrane. Invest Ophthalmol Vis Sci. 2002;43(2):458-65.

18. Gabel VP. Die Lichtabsorption am Augenhintergrund. Habilitation. Munich: Ludwig MaximiliansUniversität München; 1976.

19. Anderson RR, Parrish JA. Selective photothermolysis: precise microsurgery by selective absorption of pulsed laser radiation. Science. 1983;220:524-7.

20. Schmidt SY, Peisch RD. Melanin concentration in normal human retinal pigment epithelium. Regional variation and age-related reduction. Invest Ophthalmol Vis Sci. 1986;27(7):1063-7.

21. Roider J, et al. Microphotocoagulation: selective effects of repetitive short laser pulses. Proc Natl Acad Sci U S A. 1993;90:8463-647.

22. Brinkmann R, et al. Origin of retinal pigment epithelium cell damage by pulsed laser irradiance in the nanosecond to microsecond time regimen. Lasers Surg Med. 2000;27(5):451-64.

23. Brinkmann R, et al. Targeting the retinal pigment epithelium (RPE) by means of a rapidly scanned continuous wave $(\mathrm{CW})$ laser beam. Lasers Surg Med. 2003;32(4):252-64. 
24. Framme C, et al. Selective targeting of the retinal pigment epithelium in rabbit eyes with a scanning laser beam. Invest Ophthalmol Vis Sci. 2007;48(4):1782-92.

25. Framme C, et al. Comparison of threshold irradiances and online dosimetry for selective retina treatment (SRT) in patients treated with 200 nanoseconds and 1.7 microseconds laser pulses. Lasers Surg Med. 2008;40(9):616-24.

26. Lin CP, et al. Selective cell killing by microparticle absorption of pulsed laser radiation. IEEE J Select Topics Quantum Electron. 1999;5(4):963-8.

27. Neumann J, Brinkmann R. Boiling nucleation on melanosomes and microbeads transiently heated by nanosecond and microsecond laser pulses. J Biomed Optics. 2005;10(2):024001.

28. Neumann J, Brinkmann R. Self-limited growth of laser-induced vapor bubbles around single microabsorbers. Appl Phys Lett. 2008;93(3):033901.

29. Neumann J, Brinkmann R. Nucleation dynamics around single microabsorbers in water heated by nanosecond laser irradiation. J Appl Phys. 2007;101(11):114701.

30. Brinkmann R, Birngruber R. Selektive retina-therapie (SRT). Z Med Phys. 2007;17:6-22.

31. Fritz A, et al. Dynamics and detection of laser induced micro bubbles in the retinal pigment epithelium (RPE). Proc SPIE-OSA. 2007;6632:1C1-11.

32. Fritz A, et al. Dynamics of laser induced transient micro bubble clusters. Proc SPIE-OSA. 2009;7373:0D1-9.

33. Steiner P, et al. Time-resolved ultra-high resolution optical coherence tomography for real-time monitoring of selective retina therapy. Invest Ophthalmol Vis Sci. 2015;56(11):6654-62.

34. Neumann J, Brinkmann R. Cell disintegration by laser-induced transient microbubbles and its simultaneous monitoring by interferometry. J Biomed Opt. 2006;11(4):041112.

35. Framme C, et al. Threshold determinations for selective retinal pigment epithelium damage with repetitive pulsed microsecond laser systems in rabbits. Ophthalmic Surg Lasers. 2002;33(5):400-9.

36. Alt $\mathrm{C}$, et al. Monitoring intracellular cavitation during selective targeting the retinal pigment epithelium. Proc SPIE. 2003;4951:48-55.

37. Schüle G, et al. RPE damage thresholds and mechanisms for laser exposure in the $\mu$ s to ms time regimen. Invest Ophthalmol Vis Sci. 2005;46(2):714-9.

38. Yang $\mathrm{JH}$, et al. Morphologic changes in the retina after selective retina therapy. Graefes Arch Clin Exp Ophthalmol. 2016;254(6):1099-109.

39. Richert E, et al. Release of different cell mediators during retinal pigment epithelium regeneration following selective retina therapy. Invest Ophthalmol Vis Sci. 2018;59(3):1323-31.

40. Treumer F, et al. Vectorial release of matrix metalloproteinases (MMPs) from porcine RPE-choroid explants following selective retina therapy (SRT): towards slowing the macular ageing process. Exp Eye Res. 2012;97(1):63-72.

41. Kim HD, et al. Functional evaluation using multifocal electroretinogram after selective retina therapy with a microsecond-pulsed laser. Invest Ophthalmol Vis Sci. 2015;56(1):122-31.

42. Park YG, et al. A comparative study of retinal function in rabbits after panretinal selective retina therapy versus conventional panretinal photocoagulation. J Ophthalmol. 2015;2015:247259.

43. Han JW, et al. Comparison of the neuroinflammatory responses to selective retina therapy and continuous wave laser photocoagulation. Graefes Arch Clin Exp Ophthalmol. 2018;256(2):341-53.

44. Hussain AA, et al. Disturbed matrix metalloproteinase activity of Bruch's membrane in age-related macular degeneration. Invest Ophthalmol Vis Sci. 2011;52(7):4459-66.

45. Roider J, et al. Retinal sparing by selective retinal pigment epithelial photocoagulation. Arch Ophthalmol. 1999;117(8):1028-34.

46. Roider J, et al. Subthreshold (retinal pigment epithelium) photocoagulation in macular diseases: a pilot study. Br J Ophthalmol. 2000;84(1):40-7.

47. Brinkmann R, Roider J, Birngruber R. Selective retina therapy (SRT): a review on methods, techniques, preclinical, and first clinical results. Bull Soc Belge Ophtalmol. 2006;302:51-69.

48. Kang S, et al. Selective retina therapy in patients with chronic central serous chorioretinopathy: a pilot study. Medicine. 2016;95(3):e2524.

49. Boettner E, Wolter J. Transmission of the ocular media. Investig Ophthalmol. 1962;1(6):776-83.

50. Brinkmann R, et al. Real-time temperature determination during retinal photocoagulation on patients. J Biomed Opt. 2012;17(6):061219.

51. Framme C, et al. Structural changes of the retina after conventional laser photocoagulation and selective retina treatment (SRT) in spectral domain OCT. Curr Eye Res. 2009;34(7):568-79.

52. Roider J, et al. Selective retina therapy (SRT) for clinically significant diabetic macular edema. Graefes Arch Clin Exp Ophthalmol. 2010;248(9): 1263-72.

53. Kracht D, Brinkmann R. Green Q-switched microsecond laser pulses by overcoupled intracavity second harmonic generation. Opt Commun. 2004;231:319-24.

54. Park YG, et al. Safety and efficacy of selective retina therapy (SRT) for the treatment of diabetic macular edema in Korean patients. Graefes Arch Clin Exp Ophthalmol. 2016;254(9):1703-13.

55. Roider J, et al. Clinical results after subthreshold (RPE) photocoagulation in macular diseases. Biomedical Optics, OSA Technical Digest. 1999:11-13.

56. Prahs P, et al. Selective retina therapy (SRT) in patients with geographic atrophy due to age-related macular degeneration. Graefes Arch Clin Exp Ophthalmol. 2010;248(5):651-8. 
57. Elsner $\mathrm{H}$, et al. Selective retina therapy in patients with central serous chorioretinopathy. Graefes Arch Clin Exp Ophthalmol. 2006;244(12):1638-45.

58. Klatt C, et al. Selective retina therapy for acute central serous chorioretinopathy. Br J Ophthalmol. 2011;95(1):83-8.

59. Yasui A, et al. Retinal sensitivity after selective retina therapy (SRT) on patients with central serous chorioretinopathy. Graefes Arch Clin Exp Ophthalmol. 2017;255(2):243-54.

60. Lavinsky D, Palanker D. Nondamaging photothermal therapy for the retina: initial clinical experience with chronic central serous retinopathy. Retina (Philadelphia, Pa). 2015;35(2):213-22.

61. Framme C, et al. Selective retina therapy in acute and chronic-recurrent central serous chorioretinopathy. Ophthalmologica. 2015;234(4):177-88.

62. Guymer RH, et al. Sub-threshold nanosecond laser intervention in age-related macular degeneration: the LEAD randomized controlled clinical trial. Ophthalmology. 2019;126(6):829-38.

63. Schüle G, et al. Optoacoustic real-time dosimetry for selective retina treatment. J Biomed Opt. 2005;10(6):064022.

64. Seifert E, et al. Selective retina therapy (SRT): towards an optically controlled automatic dosing. J Biomed Opt. 2018;23(11):1-12.

65. Roider J, Brinkmann R, Birngruber R. Selective retinal pigment epithelium laser treatment - theoretical and clinical aspects. In: Fankhauser F, Kwasniewska $\mathrm{S}$, editors. Lasers in ophthalmology - basic, diagnostic and surgical aspects. The Hague: Kugler Publications; 2003. p. 119-29.

66. Roider J, et al. Therapeutical bandwidth or selective RPE-photocoagulation treatment by repetitive mu s-laser pulses $(527 \mathrm{~nm})$ - first clinical results. Invest Ophthalmol Vis Sci. 1997;38(4):404.

67. Roider J, et al. Variability of RPE reaction in two cases after selective RPE laser effects in prophylactic treatment of drusen. Graefes Arch Clin Exp Ophthalmol. 1999;237(1):45-50.

68. Brinkmann R, Schüle G. Method and device for noninvasive regulation of temperature with radiation, especially laser radiation of biological tissue. Patent EP 1279385 B1, US 6,830,567; 2002.

69. Park Y-G, et al. Tissue response of selective retina therapy by means of a feedback-controlled energy ramping mode. Clin Exp Ophthalmol. 2014;42(9):846-55.

70. Seifert E, et al. Automatic irradiation control by an optical feedback technique for selective retina treatment (SRT) in a rabbit model. Proc OSA-SPIE. 2013;8803:031-6.

71. Park YG, et al. Selective retina therapy with automatic real-time feedback-controlled dosimetry for chronic central serous chorioretinopathy in Korean patients. Graefes Arch Clin Exp Ophthalmol. 2017;255(7):1375-83.
72. Sigrist MW. Laser generation of acoustic waves in liquids and gases. J Appl Phys. 1986;7:R83-R121.

73. Schüle G, et al. Non-invasive optoacoustic temperature determination at the fundus of the eye during laser irradiation. J Biomed Opt. 2004;9(1):173-9.

74. Neumann J, Brinkmann R. Microbubble dynamics around melanosomes irradiated with microsecond pulses. Proc SPIE. 2002;4617:180-5.

75. Neumann J, Brinkmann R. Microbubble dynamics around laser heated microparticles. Proc SPIE. 2003;5142:82-7.

76. Schüle G, et al. Optoacoustic online dosimetry during selective RPE treatment. Proc SPIE. 2004;5314:286-97.

77. Seifert E, et al. Selective retina treatment (SRT): towards an acoustically controlled automatic dosing. In preparation.

78. Stoehr H, et al. Interferometric optical online dosimetry for selective retina treatment (SRT). Proc SPIE. 2007;6632:642619.

79. Müller $\mathrm{HH}$, et al. Imaging thermal expansion and retinal tissue changes during photocoagulation by high speed OCT. Biomed Opt Express. 2012;3(5):1025-46.

80. El-Brawany M, et al. Measurement of thermal and ultrasonic properties of some biological tissues. J Med Eng Technol. 2009;33(3):249-56.

81. Hasgall P, Di Gennaro F, Baumgartner C, Neufeld E, Lloyd B, Gosselin M, et al. IT'IS Database for thermal and electromagnetic parameters of biological tissues; Database Tissue Prop. [Internet] 2018; Available from: https://itis.swiss/database.

82. Kurokawa K, Makita S, Yasuno Y. Investigation of thermal effects of photocoagulation on retinal tissue using fine-motion-sensitive dynamic optical coherence tomography. PLoS One. 2016;11(6):e0156761.

83. Goetz $\mathrm{G}$, et al. Interferometric mapping of material properties using thermal perturbation. Proc Natl Acad Sci U S A. 2018;115(11):E2499-508.

84. Bizheva K, et al. Sub-micrometer axial resolution OCT for in-vivo imaging of the cellular structure of healthy and keratoconic human corneas. Biomed Opt Express. 2017;8(2):800-12.

85. Bizheva K, et al. Optophysiology: depthresolved probing of retinal physiology with functional ultrahigh-resolution optical coherence tomography. Proc Natl Acad Sci U S A. 2006;103(13): 5066-71.

86. Steiner P. Ultra-high resolution optical coherence tomography retinal tissue monitoring for dosimetry control during selective retina therapy laser treatment. PhD thesis. Bern: Bern University; 2015.

87. Spaide RF, Fujimoto JG, Waheed NK. Image artifacts in optical coherence angiography. Retina (Philadelphia, PA). 2015;35(11):2163.

88. Kaufmann D, et al. Selective retina therapy enhanced with optical coherence tomography for dosimetry 
control and monitoring: a proof of concept study. Biomed Opt Express. 2018;9(7):3320-34.

89. Roh YJ, et al. Selective retina treatment (SRT) automatically controlled by a real-time reflectometry in a rabbit model. Invest Ophthalmol Vis Sci. 2013;54(15):4136.

90. Steiner P, et al. Retinal laser lesion visibility in simultaneous ultra-high axial resolution optical coherence tomography. IEEE Photon J. 2014;6(6):1-11.

91. Kaufmann D. Combining SRT with OCT for automated dose-control [Master Thesis]. Biel, Bern: Universität Bern und Berner Fachhochschule; 2016.

92. Martinez-Conde S, Otero-Millan J, Macknik SL. The impact of microsaccades on vision: towards a unified theory of saccadic function. Nat Rev Neurosci. 2013;14(2):83.
93. Zbinden S, et al. Automatic assessment of timeresolved OCT images for selective retina therapy. Int J Comput Assist Radiol Surg. 2016;11(6):863-71.

94. Fountoukidou T, et al. Motion-invariant SRT treatment detection from direct M-scan OCT imaging. Int J Comput Assist Radiol Surg. 2018;13(5): 683-91.

95. Mooser M, et al. Quantitative, simultaneous \& collinear eye-tracked, high dynamic range optical coherence tomography at 850 and $1060 \mathrm{~nm}$. In: European Conference on Biomedical Optics. Optical Society of America; 2017.

96. Burri C. Optical coherence tomography controlled selective retina therapy for in-vivo treatment [Master Thesis]. Biel, Bern: Bern University of Applied Sciences; 2018.

Open Access This chapter is licensed under the terms of the Creative Commons Attribution 4.0 International License (http://creativecommons.org/licenses/by/4.0/), which permits use, sharing, adaptation, distribution and reproduction in any medium or format, as long as you give appropriate credit to the original author(s) and the source, provide a link to the Creative Commons license and indicate if changes were made.

The images or other third party material in this chapter are included in the chapter's Creative Commons license, unless indicated otherwise in a credit line to the material. If material is not included in the chapter's Creative Commons license and your intended use is not permitted by statutory regulation or exceeds the permitted use, you will need to obtain permission directly from the copyright holder.

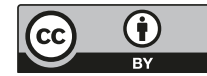

